\title{
Valveless Fluid Pumping via Zero-Net-Momentum Injection
}

\author{
Johnathan Cace \\ Department of Biomedical Engineering and Mechanics, Virginia Tech, Blacksburg, VA \\ bttps://doi.org/10.33697/ajur.2019.006 \\ Student:jcace@vt.edu \\ Mentor:staplesa@vt.edu
}

\begin{abstract}
A novel mechanical method to valvelessly pump fluid has been developed using zero-net-momentum injection via a syringe and a tilted canister that function together as a periodic mass source and sink. Unlike previously discovered valveless pumping methods, this method does not require any elastic tubing and can be achieved by simple manual actuation, making it a simpler and less expensive valveless alternative. The flow rate is highly dependent on the frequency at which momentum is injected and retracted from the system. The direction of the flow can be changed by switching the location of the syringes. This pumping paradigm has potential applications in microfluidics where elastic channels are difficult to fabricate and valveless fluid actuation methods are preferred.
\end{abstract}

\section{KEYWORDS}

Valveless Pumping; Momentum Injection; Microfluidics; Fluid Mechanics; Windkessel Effect

\section{INTRODUCTION}

As lab-on-a-chip microfluidic devices proliferate for uses such as enzyme and DNA analysis and point-of-care medical diagnostics, the need arises for better valveless flow actuation methods. ${ }^{1}$ Valveless fluid pumping in both open and closed loop systems is currently limited to four main methods: peristaltic, ${ }^{2}$ impedance, ${ }^{1}$ contraction pumping, ${ }^{3}$ and dielectrophoresis (DEP). ${ }^{4}$ Peristaltic pumping, fluid motion by compressions and expansions of an elastic tube a traveling wave motion, and impedance pumping, the transportation of fluid by tapping a section of elastic tubing in an otherwise inelastic tube repeatedly to drive flow, are both mechanical systems which require sections of elastic tubing in order to move the fluid. Contraction pumping, fluid flow created by multiple localized, rhythmic contractions of small sections of otherwise rigid tubing of differing lengths and amplitudes, has been seen in physiological systems such as the human duodenum and insects. ${ }^{6}$ For micro- and nanofluidic devices, the smaller the scale, the harder and more expensive it is to manufacture sections of elastic or locally collapsible yet rigid materials, even as 3-D printing becomes increasingly ubiquitous. ${ }^{5}$

DEP is achieved by applying an electric field in the intended direction of fluid flow and does not require the elastic or locally collapsible materials needed for peristaltic, impedance or contraction pumping. Unfortunately, DEP has a number of other drawbacks, particularly if the material is not chemically inert. The fluid can undergo electrothermal flow from high temperatures and voltages. Electrolysis can also occur, which leads to gas evolution within the fluid, causing the pump to become drastically less efficient. ${ }^{7}$ These effects can not only influence results but also limit what fluids can be pumped by DEP and the flow rates at which they can be pumped.

This paper presents a new paradigm for fluid pumping that does not require any elastic deformation of the membrane or an electric field applied to the fluid. Instead, fluid is pumped by introducing momentum into the system similar to the one introduced by Aboelkassem and Staples. ${ }^{8-12}$ That pumping mechanism works by combining principles of impedance and contraction pumping to introduce momentum into the system using multiple localized, rhythmic contractions of elastic sections of an otherwise rigid tube. These elastic sections are actuated with a phase lag and are of different sizes and amplitudes which changes the otherwise symmetric hydraulic resistance in one direction and the fluid consequently takes the path of least resistance.

The pumping mechanism introduced in this paper generates a similar pattern of asymmetric hydraulic resistance without the need for any elastic or collapsible sections of tubing. Here fluid is inserted to and retracted from the system using a syringe and a partially tilted canister that function together as a periodic mass source and sink. The syringe is filled to the plunger while the canister is filled with the fluid as well as air. The syringe is actuated with a simple sinusoidal frequency but the tilt and air in the canister, hereby referred to as the Windkessel, ${ }^{13}$ creates an asymmetry in the hydraulic resistance of the system which generates a net unidirectional 
flow to be generated. This flow is generated despite the Windkessel remaining stationary throughout experimentation and zero-netmomentum being added to the system over one period of actuation. The actuation of the syringe can be done manually, which makes the paradigm potentially useful for inexpensive or emergency medical testing. This paper investigates and quantifies the effects that the actuation frequency of the zero-net-momentum injection has on the net flow rate in a single configuration as well as the paradigm's limitations.

\section{METHODS AND PROCEDURES}

To conduct this experiment, a closed, circular system of a constant diameter was created from a $1.5 \mathrm{~m}$ long piece of high-density polyethylene (HDPE) tube with an inner diameter of $6.35 \mathrm{~mm}$ and an outer diameter of $9.525 \mathrm{~mm}$. Two $3.175 \mathrm{~mm}$ diameter holes were drilled into it $85.725 \mathrm{~mm}$ apart, in the axial direction for the syringe and Windkessel to be inserted. The ends of the tube were joined together by sliding another piece of HDPE tubing with an inner diameter of $9.525 \mathrm{~mm}$ over the ends of the primary tube, being sure to clamp it down using ties to prevent leaking. The tube was then completely filled with tap water, ensuring the removal of any air bubbles prior to experimentation.

Two identical $22 \mathrm{~mL}$ syringes were used. One was modified to make a Windkessel by removing the plunger. The syringe was filled to the plunger with $10 \mathrm{~mL}$ of tap water up with the top of the plunger attached to a Cool Muscle CM1-C-23L20C Servo Motor which allowed the syringe to be linearly actuated in a sinusoidal fashion at a set frequency. The Windkessel was filled with $4 \mathrm{~mL}$ of water and $18 \mathrm{~mL}$ of air at room temperature and atmospheric pressure. The tilt angle, $\theta$, was held constant at approximately 5 degrees from the perpendicular as seen in Figure 1.

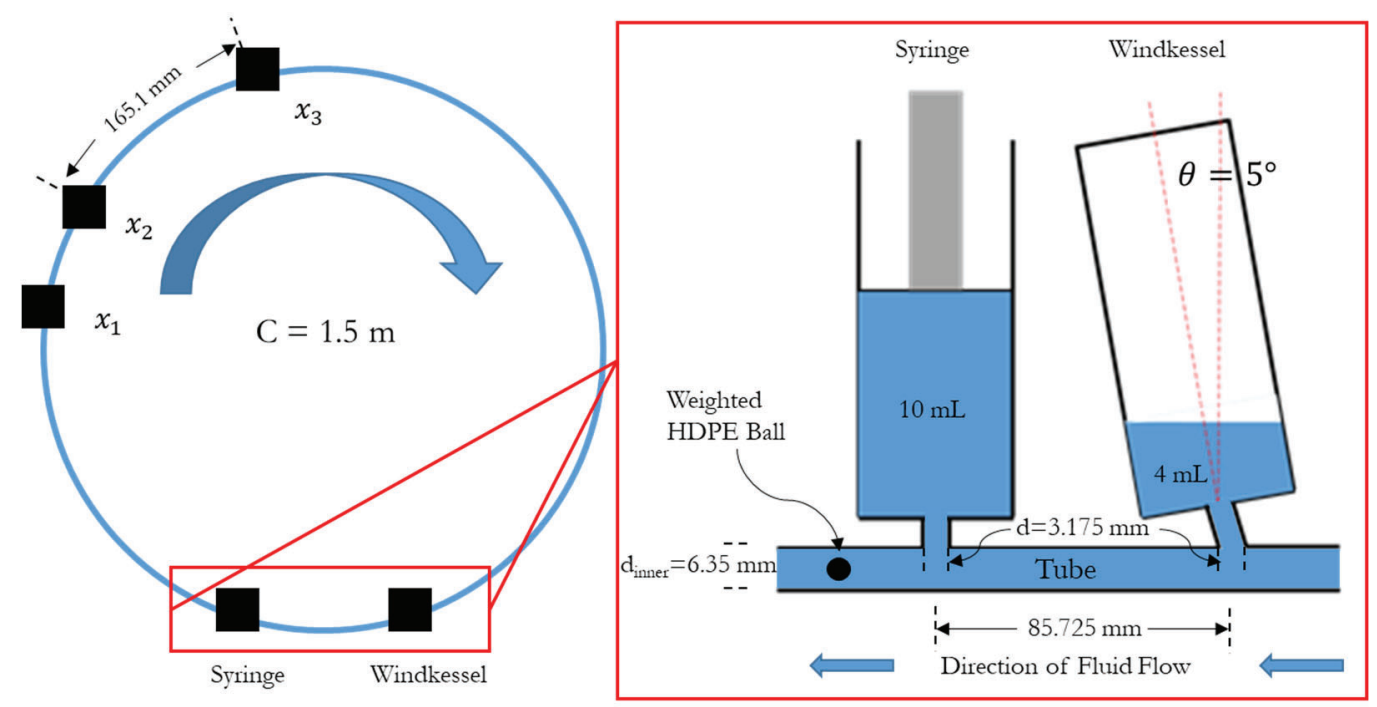

Figure 1. A generalized schematic of the experimental setup from a top view with an insert of the syringe and Windkessel from a front view. The circumference, $\mathrm{C}$, the tilt angle, $\theta$, and the most measurements have been noted as well as the direction of the fluid flow in the blue arrows. The $\mathrm{x}_{\mathrm{i}}$ points indicate the set locations for the HDPE ball for measurements with $\mathrm{x}_{1}$ being the start of actuation, $\mathrm{x}_{2}$ being the start of timing, and $\mathrm{x}_{3}$ being the end of timing.

To measure the net volumetric flow, $Q$, flowrate in the tube, knowledge was required about the time it takes to travel a known distance in the tube. An HDPE ball with a $3.175 \mathrm{~mm}$ diameter, weighted with a small metal pin to make it magnetic and as close to neutrally buoyant as possible, was inserted into the tube. It was then moved to $x_{1}$ at the start of each test using a magnet. The Servo Motor then actuated the syringe to begin the fluid flow, which reached its steady state after a few periods of actuation. To compensate for this, $x_{2}$ and $x_{3}$ were marked on the tube $165.1 \mathrm{~mm}$ apart and the time it took for the HDPE ball to go from $x_{2}$ and $x_{3}$ was manually measured. From this time and measured distances, the net volumetric flow rate was calculated as

$$
Q=\frac{0.1651 \pi r^{2}}{t}
$$

Equation 1.

where $r$ is the radius of the tube and $t$ is the time. The actuation began with the injection phase during which $5 \mathrm{~mL}$ of water was injected into the system forcing the same volume of water to enter the Windkessel at different frequencies. Data was collected starting at a frequency $5 / 6 \mathrm{~Hz}$ and increased in increments of $1 / 6 \mathrm{~Hz}$ up to $13 / 6 \mathrm{~Hz}$. If an air bubble got into system, the tube 
would get refilled, and the data was ignored from all prior tests until one set of data could be recorded from $5 / 6$ to $13 / 6 \mathrm{~Hz}$ under the same conditions. Upon completion of one set, the system was refilled to maintain continuity between data sets.

The retraction phase began once the syringe reached $5 \mathrm{~mL}$ of water output, causing $5 \mathrm{~mL}$ of water being immediately drawn back into the syringe from the Windkessel. The periodicity of this momentum injection and retraction introduces no net-momentum to the system over time despite the observed net-unidirectional flow of the fluid. A diagram of the syringe and actuation over one period is shown in Figure 2.
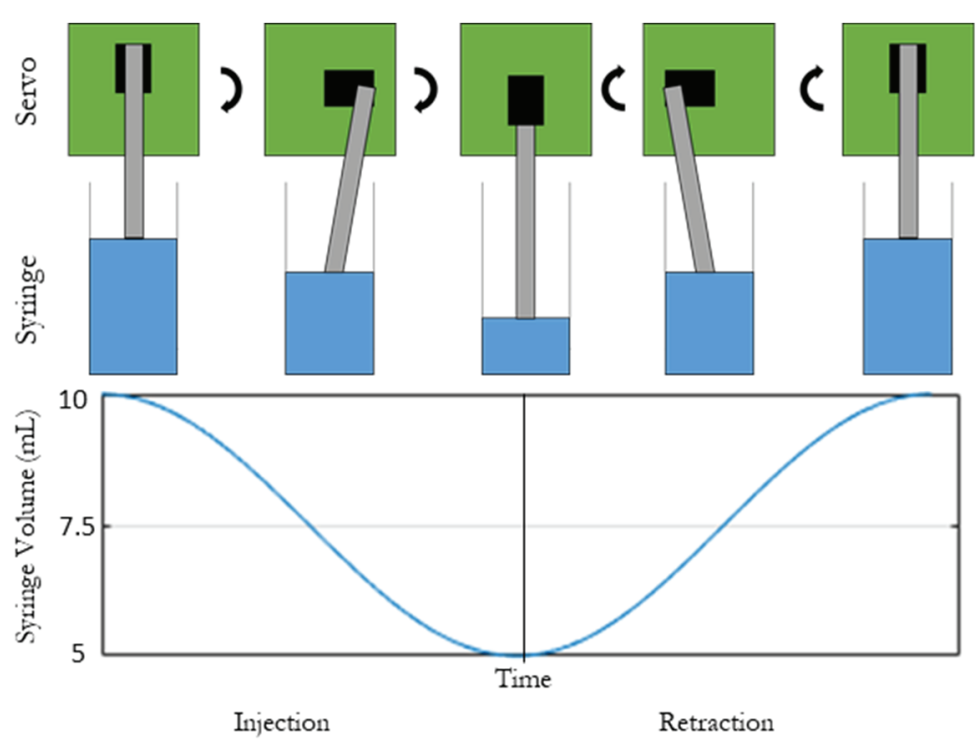

Figure 2. Injection and retraction phases with corresponding syringe and Servo Motor positions.

\section{RESULTS}

The experiments showed that a net flow could be generated in a closed system by injecting momentum into the fluid via this zeronet-momentum injection principle. It also showed that the flow rate is highly dependent on the frequency at which momentum is injected. Figure $\mathbf{3}$ shows the net volumetric flow rate versus the frequency at which momentum was injected. For each frequency of injection, six data sets were generated.

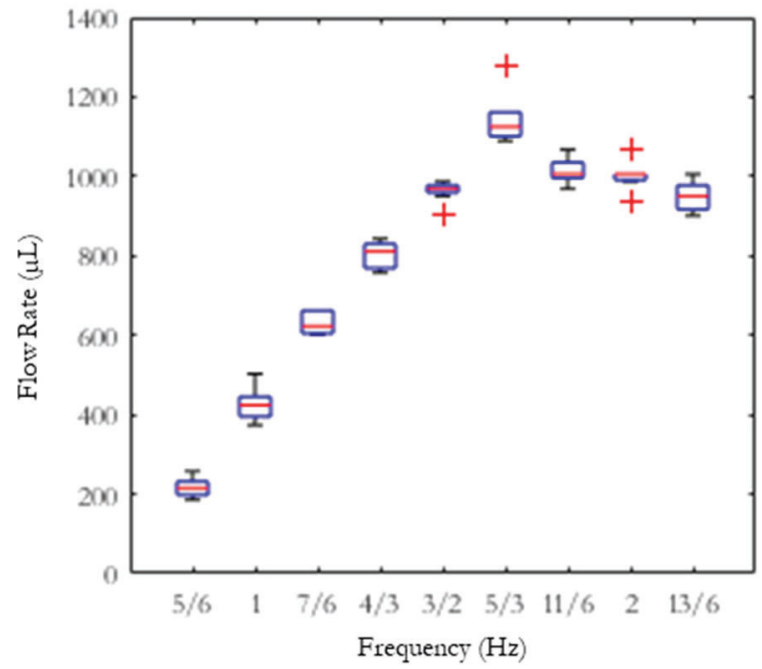

Figure 3. A plot of all of the measured flow rates with respect to the pumping frequency with boxplots of the data for each frequency. 
It is important to note that despite the measured net flow, the instantaneous flow rate of the fluid was not constant over time but varied periodically with a mean net flow component. During the injection phase of the pumping, flow was generated in the direction of the net flow. During the retraction phase, however, flow was generated in the opposite direction of the net flow but with a much smaller magnitude. As the frequency increased, the magnitude of the reversed flow during the retraction phase decreased, approaching approximately zero. Swapping the locations of the two syringes as well as changing the Windkessel tilt angle $\theta$ to a negative value changed the direction but not the magnitude of the resulting flow.

\section{DISCUSSION}

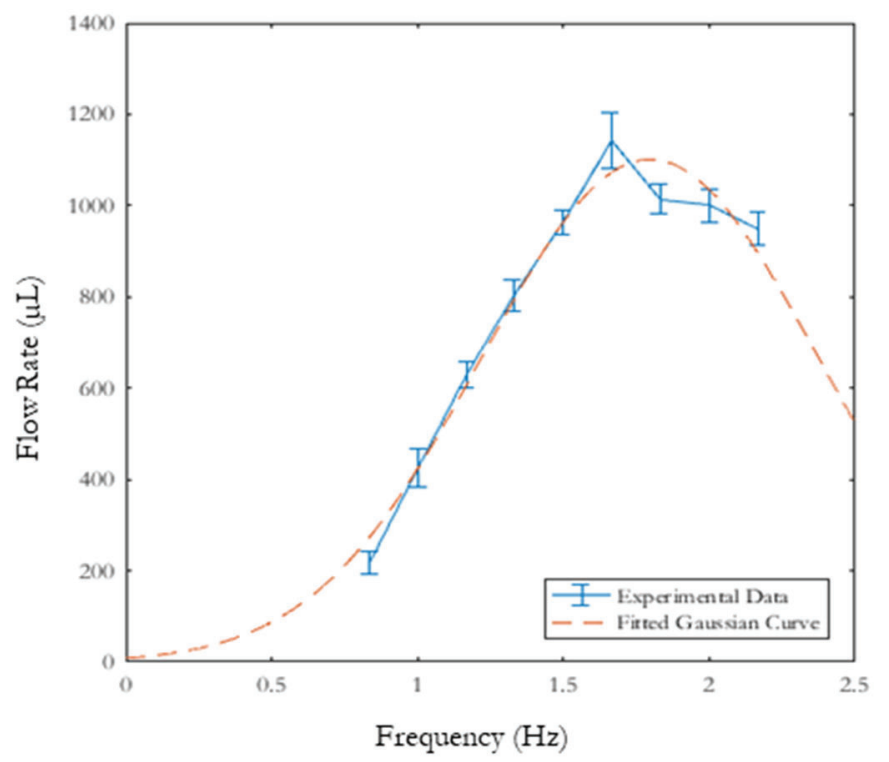

Figure 4. A plot of all of the tests completed with error bars and a fitted Gaussian curve, relating flow rate to the pumping frequency.

The flow rate reached a maximum at $5 / 3 \mathrm{~Hz}$ and then began to decrease showing that there is an optimum actuation frequency that maximizes the flow rate. This result is similar to the theoretical results found in Aboelkassem and Staples ${ }^{8-12}$ for a related actuation method where instead of injecting and retracting momentum via fluid, the tube wall collapses and retracts in a zero-netmomentum-injection manner to drive a flow. In those papers, it is established that there is an optimum actuation frequency for that pumping mechanism. A Gaussian curve was fitted to the data and it predicts a maximum flow rate of $1101 \mu \mathrm{L} / \mathrm{s}$ at a frequency of $1.797 \mathrm{~Hz}$. The fitted curve has an R-squared value of 0.974 and is plotted with the collected data in Figure 4. A Gaussian model was chosen for simplicity since it is everywhere positive and has a single peak like the data. It also serves as a useful experimental comparison to the theoretical work of Aboelkassem and Staples.

To fully understand this pumping mechanism, many more experimental configurations should be completed including but not limited to changing the size of the tube, the fluid being pumped, the tilt angle of the Windkessel and the volumes of fluid in both the syringe and Windkessel. Without this data, stating the scope and scalability of this pumping with full certainty is impossible. However, comparing the presented experimental results to the theoretical work of Aboelkassem and Staples as well as the instances in nature where similar pumping has been demonstrated to exist ${ }^{6,8-12}$, it can reasonably assumed that the pumping for incompressible viscous flow at low Reynolds number $(\operatorname{Re} \sim \delta)$ can be scaled for channels with a length that is much greater than its width $(\delta=$ $\mathrm{W} / \mathrm{L}<<1$ ). For this experiment, the width is the diameter of the tube with the length being the circumference which yields a Reynolds number of 0.00423.

There were two primary sources of error in this experiment. The first is the tilt angle of the Windkessel. While a wedge was used to keep the Windkessel at five degrees during every experiment, the pressures built up inside the system from the higher frequency tests required a lot more force on the Windkessel to hold it in place. This friction caused the hole to deform slightly over time, creating some uncertainty about the exact tilt angle of the Windkessel. This issue could be resolved in future work by using a less deformable material than HDPE for the tubing but an inexpensive, rigid and at least semitransparent material was needed to properly measure fluid flow. Once this zero-net-momentum injection pump is better understood, a stronger and more opaque tube can be used with the same success. 
The second source of error was with air bubbles entering the system. These air bubbles would either dramatically reduce the flow or eliminate it entirely depending on the number of bubbles and their size. Although every effort was made to minimize this effect, some smaller air bubbles could not be removed despite the refilling procedure and may have influenced the measured flow rate.

\section{CONCLUSIONS}

This paper has investigated a new paradigm in valveless fluid pumping which uses a zero-net-mass injection principle and a periodic mass source and sink to drive a flow in a circular cross section channel. Based on the results presented in Figure $\mathbf{3}$, it is clear that this newly developed method of fluid pumping is successful in creating a net unidirectional flow. Furthermore, by switching the locations of the syringe and Windkessel, the direction of flow could also be changed. Based on the net volumetric flow rate data, a general trend can be observed between the frequency of momentum injection and the volumetric flow rate as seen in Figure 4 . The flow rate increased as the frequency increased up to a maximum at around $5 / 6 \mathrm{~Hz}$ and then decreased at higher frequencies, which follows the trend found in similar pumping mechanisms ${ }^{8-12}$ with a predicted maximum flow rate of $1101 \mu \mathrm{L} / \mathrm{s}$ at a frequency of $1.797 \mathrm{~Hz}$.

One advantage of this pumping mechanism over other valveless pumping mechanisms for future microscale fluid manipulation applications $^{1-5}$ is that it does not require the use of sections of hard-to-fabricate elastic tubing, or electric current which can change the chemical properties of the fluid. Additionally, this actuation mechanism can be easily implemented manually, although the results presented were done by a computer-controlled Servo Motor for accuracy. These advantages open up the possibility for pumping fluid cheaply and robustly in a wide variety of applications, particularly in microfluidics where lab-on-a-chip technology is becoming ubiquitous.

\section{ACKNOWLEDGMENTS}

The author thanks Dr. Anne Staples for overseeing this project and her mentorship, guidance and support in turning this abstract concept into a reality. He also thanks Ethan Compton, Andrew Nicklow, Steven Catapano, Timothy Dundorf, and Grace Ma for assisting in data collection and experimental design.

\section{REFERENCES}

1. Rinderknecht, D., Hickerson, A. I., Gharib, M. (2005) A valveless micro impedance pump driven by electromagnetic actuation. Journal of Micromechanics and Microengineering 15(4):861-866.

2. Jaffrin, M.Y., Shapiro, A.H. (1971) Peristaltic Pumping. Annual Review of Fluid Mechanics 3(1):13-37.

3. Macagno, E.O., Christensen, J. (1980) Fluid Mechanics of the Duodenum. Annual Review of Fluid Mechanics 12(1):139-158.

4. Mauro, A. (1980) Dielectrophoresis: The Behavior of Neutral Matter in Nonuniform Electric Fields. Herbert A. Pohl. The Quarterly Review of Biology 55(1):68-69.

5. Kirby, B.J. (2013) Micro- and nanoscale fluid mechanics: transport in microfluidic devices (Cambridge University Press, Cambridge).

6. Socha, J.J., Lee, W., Harrison, J., Waters, J., Fezzaa, K., Westneat, M. (2008) Correlated patterns of tracheal compression and convective gas exchange in a carabid beetle. Journal of Experimental Biology 211(21):3409-3420.

7. Zellner, P., Agah, M. (2012) Silicon insulator-based dielectrophoresis devices for minimized heating effects. Electrophoresis 33(16):2498-2507.

8. Aboelkassem, Y., Staples, A.E. (2011) Flow transport in a microchannel induced by moving wall contractions: a novel micropumping mechanism. Acta Mechanica 223(3):463-480.

9. Aboelkassem, Y., Staples, A.E. (2013) Selective pumping in a network: insect-style microscale flow transport. Bioinspiration \& Biomimetics 8(2):026004.

10. Aboelkassem, Y., Staples A.E. (2012) Stokeslets-meshfree computations and theory for flow in a collapsible microchannel. Theoretical and Computational Fluid Dynamics 27(5):681-700.

11. Aboelkassem, Y., Staples, A.E., Socha J. J. (2011) Microscale Flow Pumping Inspired by Rhythmic Tracheal Compressions in Insects. ASME 2011 Pressure Vessels and Piping Conference: Volume 4. doi:10.1115/pup2011-57061

12. Aboelkassem, Y., Staples, A.E. (2013) A bioinspired pumping model for flow in a microtube with rhythmic wall contractions. Journal of Fluids and Structures 42:187-204.

13. Sagawa, K., Lie, R., and Schaefer, J. (1990) "Translation of Otto frank's paper "Die Grundform des arteriellen Pulses" zeitschrift für biologie 37: 483-526 (1899)." Journal of Molecular and Cellular Cardiology 22, 3, 253-254. 


\section{ABOUT STUDENT AUTHOR}

Johnathan Cace completed this work while studying Engineering Science and Mechanics at Virginia Tech. Johnathan intends to graduate in 2020 and continue doing research in the area of fluid mechanics.

\section{PRESS SUMMARY}

Pumping fluids at the microscale have become increasingly important as lab-on-a-chip technology continues to advance.

Unfortunately, the existing valveless pumping mechanisms to drive flows at the microscale require internal moving parts or elastic tubing which are both expensive and prone to malfunction. A novel mechanical method to valvelessly pump fluid has been developed via a syringe and a tilted canister that function together to generate a unidirectional flow by periodically injecting and retracting momentum via mass. Experiments involving this pump show that the generated flow rate depends on the frequency of the syringe actuation. This pumping mechanism does not use any internal moving parts or elastic tubing and can even be achieved by simple manual actuation which could have implications in low-cost devices for global health applications. 